Chair for Management Science and Energy Economics University of Duisburg-Essen

EWL Working Paper No. 06/12

\title{
A Hotelling Model for Fixed-Cost Driven Power Generation
}

by

Andreas A. Renz

and

Christoph Weber

January 2013 


\title{
A HOTELLING MODEL FOR FIXED-COST DRIVEN POWER GENERATION
}

by Andreas A. Renz and Christoph Weber

\begin{abstract}
This paper links Hotelling's theory, in recent literature applied to an emission constrained environment, with the classical capacity planning framework to describe portfolio time-paths in electricity production. Emission targets are considered by a ceiling on the stock of pollution. We propose conditions for an efficient production portfolio as a subset of available technologies. We then derive potential production portfolio time-paths for a renewable, a fossil and a carbon capturing technology that differ according to their fixed and variable costs, their efficiency and their polluting characteristics. We conclude that the share of the fossil technology will continuously decrease, the scarce resource will be fully exploited. On each constrained path, the stock of pollution will remain at the ceiling for a non-zero time period. Emission targets push down scarcity rents, an option for carbon capturing would decrease societal costs and uphold scarcity rents.
\end{abstract}

Keywords : Scarce resources, Optimal control theory, Hotelling, Valuation, Non-renewable resource, Pollution target, Climate change, Peak-load-pricing

JEL-Classification : C61, Q32, Q41, L94

DIPL.-WI.-ING. ANDREAS A. RENZ

Chair for Management Sciences and Energy Economics, University of Duisburg-Essen (Campus Essen)

Universitätsstr. 11, 45117 Essen

++49 - (0)2 01 / 183-2399

www.ewl.wiwi.uni-due.de

andreas.renz@gmail.com
PROF. DR. CHRISTOPH WEBER

Chair for Management Sciences and Energy Economics, University of Duisburg-Essen (Campus Essen)

Universitätsstr. 11, 45117 Essen

++49 - (0)2 01 / 183-2966

www.ewl.wiwi.uni-due.de

christoph.weber@uni-due.de

The authors are solely responsible for the contents which do not necessarily represent the opinion of the Chair for Management Sciences and Energy Economics. 


\section{Introduction}

While the need for a global reduction of greenhouse gas emissions is widely accepted, the actual implementation process within individual countries recently stagnated. One reason lies in the high sensitivity of national economies towards energy prices. And since the energy supply strongly relies on the combustion of fossil fuels, lower emissions mostly mean also higher energy costs. There is also consensus that there is no alternative to eventually shift from scarce fuels towards a sustainable energy supply in the (very) long run. But the point of time and the way raises controversy.

Before global warming has become a centerpiece of political decision making, the main challenge in the energy markets were the limited reserves of coal, gas and oil. Hotelling (1931) first described the existence of a rent leading to price increases of resources to account for their scarcity. This holds true for both monopolistic structures and under free competition, (Weinstein and Zeckhauser, 1975; Stiglitz, 1976).

Recently, Hotelling's work has undergone a renaissance now considering targets for reduction of greenhouse gas emissions, (Tahvonen, 1997; Chakravorty et al., 2006; Smulders and van der Werf, 2008; Chakravorty et al., 2008; Lafforgue et al., 2008). To account for $\mathrm{CO}_{2}$-reduction efforts (e.g., aiming for $550 \mathrm{ppm}$ as discussed by Stern (2007)), Chakravorty et al. (2006) introduce a carbon ceiling which limits the absolute amount of $\mathrm{CO}_{2}$ in the atmosphere. They show the optimal extraction paths for energy production with coal, solar and a potential $\mathrm{CO}_{2}$-abatement under different demand trends. They conclude that in all cases the stock of $\mathrm{CO}_{2}$ is build up using solely coal. At the ceiling the natural dilution and anthropogenic $\mathrm{CO}_{2}$-emissions are balanced and multiple mixes are possible. The fossil fuel is then used until exploitation.

Chakravorty et al. (2008) also illustrate the extraction paths of coal and gas competing with a renewable technology. Neglecting any unit cost, the different polluting characteristics and scarcities of both fossil fuels are the only differentiator to satisfy a constant, price-elastic demand. In traditional Hotelling models where multiple scarce resources are competing, the rule "least cost first" applies (cf. Herfindahl (1967)). Chakravorty et al. (2008) show that this principle of using the "better" resource first may be reversed. Due to a natural dilution rate proportional to the absolute amount of $\mathrm{CO}_{2}$ in the atmosphere, the "bad", more polluting resource may be used first if gas is not abundant. Additionally, a switching from dirty coal to clean gas and back to coal is possible. This effect had not been observed in earlier Hotelling literature. Smulders and van der Werf (2008) show that if the assumption of perfect substitution is released not only carbon content, but also productivity determines an efficient energy mix. All models mentioned above assess energy use from a general primary energy perspective. Though, the characteristics of different energy using sectors differ heavily.

Electricity production, followed by transportation is the largest consumer of fossil fuels and consequently emitter of greenhouse gases. Comparing both sectors shows that the effect of rising energy prices and reduction efforts has been quite different. Efficiency increases in Transportation have been a result of mainly cost pressure through high energy prices where relative emission reductions come as a side effect. But oil has stayed the primary energy source until today. In contrast, tight emission targets have led to a shift in the mix of electricity production in certain countries. E.g., in Germany, emission targets have led to a share of renewables in the electricity production of almost $20 \%$ and this share is still increasing, (AG Energiebilanzen e.V., 2012). But 
this development was triggered by guaranteed feed-in tariffs, not by a functioning emission market. An extension of existent Hotelling models to electricity production should contribute to a better understanding how to implement appropriate policies.

Given the non-storability of electricity, power generation portfolios typically include a diversified mix of assets with varying shares of fixed and variable cost components (e.g. coal and gas power plants). ${ }^{1}$ To account for this fact, we assess the development of an efficient electricity production portfolio in the context of a scarce polluting and a renewable technology considering both variable and fixed costs as well as differences in productivities. To accommodate these specifics, the present paper applies the classical deterministic capacity planning or peak load pricing problem within a Hotelling framework. It thereby links existing peak load pricing theories (as described in e.g., Steiner (1957), Hirshleifer (1958), Boiteux (1960), Crew and Kleindorfer (1979), Chao (1983) and Weber (2005)), with literature using dynamic optimization to describe optimal extractions paths for a scarce resource in an emission constrained environment. At given fixed and variable costs, we derive the conditions that make a technology being part of the efficient portfolio.

This allows us to forecast the development of an electricity production portfolio depending on the size of the initial fossil stock and (possibly) an emission target. As expected, the energy mix tends towards the clean technology over time, though the resource is fully exploited at infinity. In contrast to Chakravorty et al. (2006), a carbon capturing technology may complement the portfolio already in a phase where the carbon stock is still increasing. Its share would then decrease as soon as the carbon stock has reached the maximum level. In each carbon emission constrained path a time period of non-zero length exists where the energy mix between the fossil and the renewable is stable. We further confirm intuition in the way that resource owners are likely to oppose emission targets from an pure economic perspective (where possible damage through global warming is neglected).

The remainder of this paper is structured as follows: First, we define the characteristics to describe the problem using peak load pricing. Then, we specify the optimization problem using optimal control theory in a very specific case. We then detail the conditions to be fulfilled for a technology to be part of the efficient portfolio and extend the problem to a more general case. In section 4, we apply the model to a case with a clean and a polluting technology and assess the impact of a possibility of carbon abatement. We show the development of the efficient portfolio over time depending on different initial resource stocks and the impact on scarcity rents. In section 5 we draw conclusions. Longer proofs and details of extraction paths are given in the Appendix.

\section{Definitions}

\subsection{Demand}

In classical peak load pricing models, demand is defined over a planning period (i.e. one year) which is divided into sections of equal length (i.e. one hour) and described by a load duration curve (ldc) as comprehensively explained in Sunderkötter and Weber (2012) and Steffen and Weber (2012). Figure 1 briefly explains the obtention of the ldc $D:[0 ; S] \mapsto \mathbb{R}_{+}, s \rightarrow D(s)$ : On the left

\footnotetext{
${ }^{1}$ Sunderkötter and Weber (2012) assess the effect of diversification on optimality conditions for efficient generation portfolios.
} 
side, the development of the demand $D_{o}(a)$ in the course of multiple consecutive days (a planning period of $S)^{2}$ is shown, on the right side the demand is rearranged in decreasing order of magnitude which forms the load duration curve. Then, $D(s)$ describes which share of total operation time $s$ per planning period, a load equal or higher than $D(s)$ is requested. ${ }^{3}$ Consider $D(s)$ to be continuously differentiable and strictly decreasing from $D(0)=D_{\max }$ to $D_{\min }$ over the interval $[0 ; \mathrm{S}]$. The ldc is assumed to be constant over time, demand to be price-inelastic. ${ }^{4}$

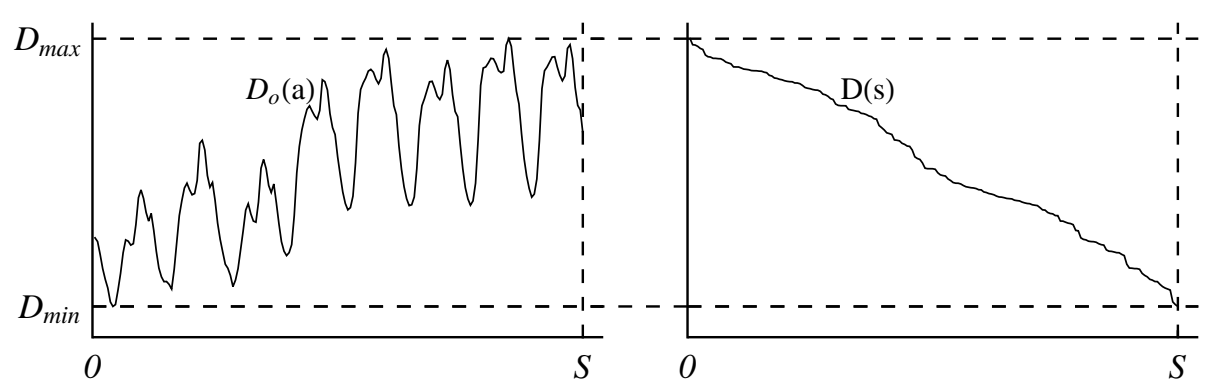

Figure 1: Chronological load demand development $D_{o}(a)$ over a defined planning period $S$ and respective load duration curve $\mathrm{D}(\mathrm{s})$ for the same planning period.

\subsection{Generation system}

The demand is met via a power plant park where technologies of similar type are clustered. Each technology $u \in A=\{1, \ldots, n\}$ is characterized by fixed costs per period $c_{i n v, u}$ and variable costs per electricity unit produced $c_{o p, u} \cdot c_{o p, u}$ includes variable operating, maintenance and extraction cost. It does not include the resource owner's scarcity rent as a result of scarce resources as well as cost of $\mathrm{CO}_{2}$-allowances as a result of emission reduction targets. These costs are derived explicitly within the model. For simplicity reasons $c_{i n v, u}$ and $c_{o p, u}$ are assumed to be constant over time.

\subsection{Scarce resources as input factors and $\mathrm{CO}_{2}$-limits}

Fuels (e.g., coal or gas) are the only input factors for electricity production. Each technology is assumed to burn only a single fuel type. Let $X_{u}^{0}$ be the initial reserve and $X_{u, t}$ the stock available of resource $u$ at time $t$. Then,

$$
\begin{array}{r}
\dot{X}_{u, t}=-x_{u, t} \text { for all } u \in A \text { and } t \in[0 ; \infty) \\
\text { with } X_{u, 0}=X_{u}^{0} .
\end{array}
$$

Converting the chemical energy of the fuels into electrical energy comes with a loss which we describe by a time-stable heat rate $h_{u}$ which is assumed to depend on the technology.

Emission reduction efforts to stabilize the $\mathrm{CO}_{2}$-content in the atmosphere (e.g., at $550 \mathrm{ppm}$ ) is accounted for through a $\mathrm{CO}_{2}$ ceiling $\bar{Z}$. Let $Z_{0}$ be the initial amount of $\mathrm{CO}_{2}$ in the atmosphere.

\footnotetext{
${ }^{2}$ Note that a representative load duration curve has to be defined over a representative period which is typically one year.

${ }^{3}$ For illustrative purposes we call the defined planning period 'year' and the share of total operating time 'operating hours' in the following.

${ }^{4}$ Bohi (2010) discusses how a peak load pricing framework can be adapted to account for an elastic demand.
} 
The actual amount of $\mathrm{CO}_{2}$ in the atmosphere $Z_{t}$ is assumed to be below or at the ceiling at each point in time. A natural dilution proportional to the absolute amount of $\mathrm{CO}_{2}$ in the atmosphere, and the specific emissions $\zeta_{u}$ when burning the fossil fuels determine the amount of $\mathrm{CO}_{2}$ in the atmosphere. So,

$$
\dot{Z}_{t}=\sum_{u=1}^{n} \zeta_{u} \cdot x_{u, t}-\alpha \cdot Z_{t} \text { with } Z_{t} \leq \bar{Z} \text { and } 0<\alpha<1
$$

\subsection{Peak load pricing}

We base our approach on the classical peak load pricing model as comprehensively described in the existing literature. Installed capacities $K_{u}$ and produced energy $Q_{u}$ per technology are thereby optimized to meet an inflexible demand $D(s)$ at minimum cost. As illustrated in figure 2, at given variable and fixed costs per technology, a technology is part of the efficient portfolio when it is costminimal (considering both fixed and variable cost) for a non-zero operating time interval within $[0 ; S]$. When the technologies of the efficient portfolio are arranged in decreasing order of fixed costs, each technology $u$ is framed by a maximum $s_{u-1, u}$ and a minimum operating time $s_{u, u+1}$. Since two different technologies cannot be optimal at the same point of operating time the upper bound of one technology has to be the lower one of another. Exceptions apply for the first and last technologies, since these are framed via the operating hours per year $S$ for the technology with highest fixed costs and zero for the technology with lowest. We define $D\left(s_{0,1}\right)=D(S)=D_{\text {min }}=0$ and $D\left(s_{n, n+1}\right)=D_{\max }=D(0)$. Consequently, we specify the efficient portfolio via a vector $\vec{s}^{*}$ describing all operating boundaries which specify the efficiency border. ${ }^{5}$

Installed capacities per technology $u$ at time $t$ are then implicitly described by

$$
K_{u, t}\left(s_{u-1, u, t}, s_{u, u+1, t}\right)=D\left(s_{u, u+1, t}\right)-D\left(s_{u-1, u, t}\right),
$$

and electricity produced by

$$
\begin{aligned}
Q_{u, t}\left(s_{u-1, u, t}, s_{u, u+1, t}\right)= & \int_{s_{u, u+1, t}}^{s_{u-1, u, t}} D(s) d s+D\left(s_{u, u+1, t}\right) \cdot s_{u, u+1, t} \\
& -D\left(s_{u-1, u, t}\right) \cdot s_{u-1, u, t}
\end{aligned}
$$

for all $u \in\{1,2, \ldots n\}, s \in[0, S]$ and $t \in[0, \infty) .{ }^{6}$ Figure 2 describes the characteristics and the interaction of technology-specific cost curves $C_{u}$ and boundaries of efficient operating time $\vec{s}^{*}$. It is important to note that whenever $\vec{s}^{*}$ is clear, the composition of energy produced per technology and installed capacities are defined implicitly. In contrast to the most classical peak load pricing models, we arrange the technologies in decreasing order of fixed costs instead of variable costs. The reason behind is that in the long-run variable costs are much more volatile than fixed costs. This allows us to keep the order consistent over time.

Beside the concept of a ldc, the economical meaning of $s_{u, u+1}$ is key for the result established

\footnotetext{
${ }^{5}$ The superscript $*$ in $\vec{s} *$ marks boundaries between two technologies (i.e. control variables) that define the efficiency border. A similar vector $\vec{s}$ characterizes all intersection of existing cost curves.

${ }^{6}$ Consequently the use of resource $u$ at time $t x_{u, t}$ equals the produced electricity $Q_{u, t}$ times the respective heat rate $h_{u}$.
} 
below. $s_{u, u+1}$ marks the borderline operating hours between the technologies $u$ and $u+1$. For operating hours $s>s_{u, u+1}$, the higher fixed cost technology $u$ is economical to use. For operating hours $s<s_{u, u+1}$, technology $u+1$ is the preferred technology.

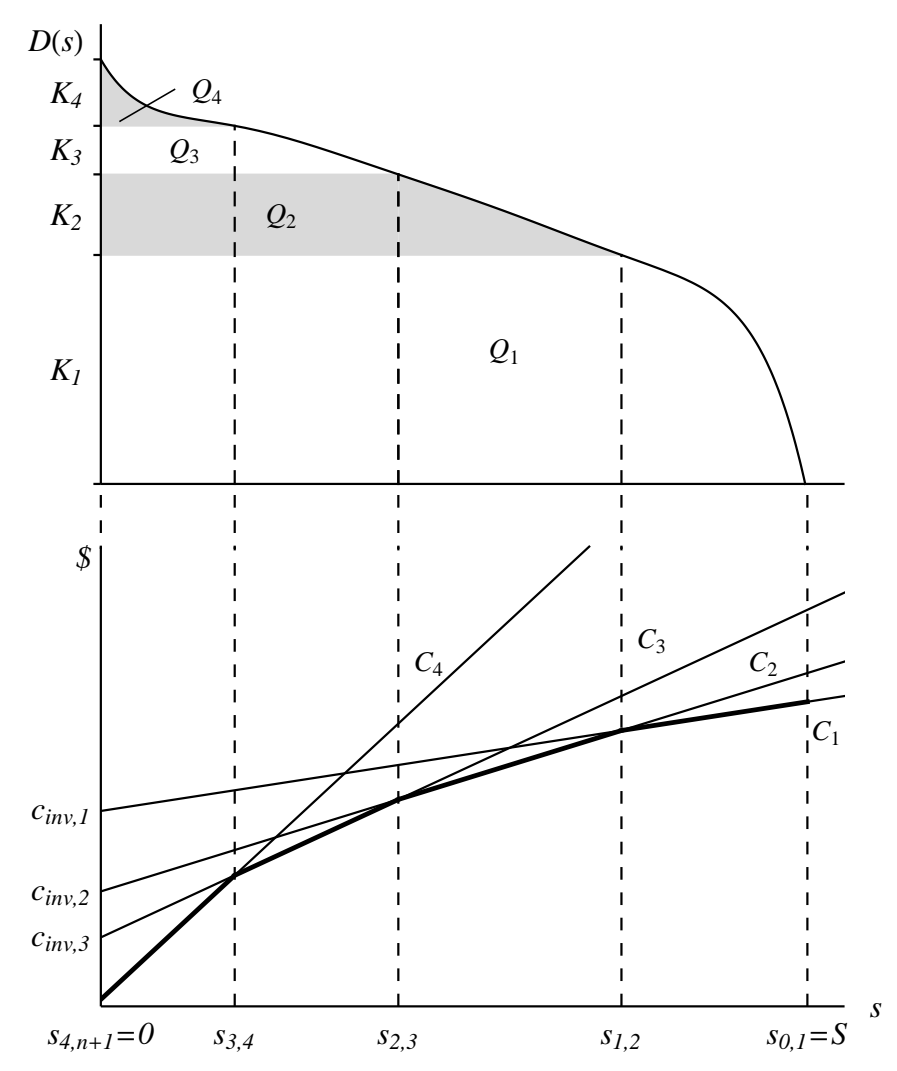

Figure 2: Formation of the efficiency border (fat line in lower graph) in a 4-technology-case and derivation of the efficient installed capacities $K_{u}$ and the energy produced per technology $Q_{u}$. Technologies are described via operating time dependent cost curves $C_{u}(s)=c_{i n v, u}+c_{o p, u} \cdot s$.

\section{Development of a Hotelling model}

\subsection{Optimal solution for a specific n-technology scenario}

Making use of optimal control theory as pioneered by L. S. Pontryagin et al. (1962) and others, it is possible to optimize the previously outlined system dynamically over time. State variables thereby describe the actual state of the system at each point of time and are influenced via control variables, of which the optimal values are to be determined. In our set-up $\vec{s}_{t}^{*}$ may be used as a vector of control variables, since it implicitly defines which capacity per technology is to be installed and consequently which amount of energy is produced at each point of time $t$. Relevant state variables are $Z_{t}$ describing the amount of $\mathrm{CO}_{2}$ in the atmosphere and $X_{u, t}$ describing the available stock at time $t$. The optimization problem is then formulated as a cost minimization problem (=maximization of negative cost) over time. For the time being we assume that all 
technologies $u$ are part of the efficient solution. With a constant discount rate $\rho$, making use of (3) and (4), the time-integral cost function to be minimized is then

$$
\begin{array}{r}
\max _{s_{u, v, t}} \int_{0}^{T}\left[-\sum_{u=1}^{n} c_{i n v, u} \cdot K_{u, t}\left(s_{u-1, u, t}, s_{u, u+1, t}\right)\right. \\
\left.-\sum_{u=1}^{n} c_{o p, u} \cdot Q_{u, t}\left(s_{u-1, u, t}, s_{u, u+1, t}\right)\right] e^{-\rho j} d t
\end{array}
$$

Subject to (1) and (2), the Lagrangian then writes

$$
\begin{aligned}
\mathcal{L}_{t}= & -\sum_{u=1}^{n} c_{i n v, u} \cdot K_{u, t}\left(s_{u-1, u, t}, s_{u, u+1, t}\right) \\
& -\sum_{u=1}^{n} c_{o p, u} \cdot Q_{u, t}\left(s_{u-1, u, t}, s_{u, u+1, t}\right) \\
& -\sum_{u=1}^{n} \lambda_{u, t} \cdot h_{u} \cdot Q_{u, t}\left(s_{u-1, u, t}, s_{u, u+1, t}\right) \\
& +\lambda_{p, t}\left[\sum_{u=1}^{n} \zeta_{u} \cdot Q_{u, t}\left(s_{u-1, u, t}, s_{u, u+1, t}\right)-\alpha \cdot Z_{t}\right] \\
& +\nu_{c, t} \cdot\left(\bar{Z}-Z_{t}\right),
\end{aligned}
$$

and first order conditions for an interior solution are as follows

$$
\begin{aligned}
\frac{\partial L_{t}}{\partial s_{u, u+1, t}}= & D_{s_{u, u+1, t}} \cdot\left(-c_{i n v, u+1, t}+c_{i n v, u, t}-c_{o p, u, t} \cdot s_{u, u+1, t}\right. \\
& +c_{o p, u+1, t} \cdot s_{u, u+1, t}-\lambda_{u, t} \cdot h_{u} \cdot s_{u, u+1, t}+\lambda_{u+1, t} \cdot h_{u+1} \cdot t_{u, u+1, t} \\
& \left.+\mu_{p, t} \cdot\left(\zeta_{u}-\zeta_{u+1}\right) \cdot t_{u, u+1, t}\right)=0 \text { for all } u \in\{1,2, \ldots, n-1\},
\end{aligned}
$$

together with the complementary slackness condition

$$
\nu_{c, t} \geq 0, \bar{Z}-Z_{t} \geq 0, \nu_{c, t}\left(\bar{Z}-Z_{t}\right)=0
$$

The dynamics of the covariates are defined by

$$
\dot{\lambda}_{u, t}=\rho \cdot \lambda_{u, t}-\frac{\partial L_{t}}{\partial X_{u, t}}=\rho \cdot \lambda_{u, t} \Rightarrow \lambda_{u, t}=\lambda_{u, 0} \cdot e^{\rho t}
$$

and

$$
\dot{\lambda}_{p, t}=\rho \cdot \lambda_{p, t}-\frac{\partial L_{t}}{\partial Z_{t}}=(\rho+\alpha) \lambda_{p, t}+\nu_{c, t}
$$

And consequently the internal solution of a cost minimal portfolio is defined as follows: 


$$
s_{u, u+1, t}=\frac{c_{i n v, u}-c_{i n v, u+1}}{\left(c_{o p, u+1}-c_{o p, u}\right)+\left(h_{u+1} \cdot \lambda_{u+1, t}-h_{u} \cdot \lambda_{u, t}\right)-\lambda_{p, t}\left(\zeta_{u+1, t}-\zeta_{u, t}\right)},
$$

or when grouping costs per technology, by

$$
s_{u, u+1, t}=\frac{c_{i n v, u}-c_{i n v, u+1}}{\left(c_{o p, u+1}+h_{u+1} \cdot \lambda_{u+1, t}-\lambda_{p, t} \cdot \zeta_{u+1, t}\right)-\left(c_{o p, u}+h_{u} \cdot \lambda_{u, t}-\lambda_{p, t} \cdot \zeta_{u, t}\right)},
$$

which allows to further combine variable cost components by

$$
c_{v a r, u, t}=c_{o p, u}+h_{u} \cdot \lambda_{u, t}-\lambda_{p, t} \cdot \zeta_{u, t}
$$

in

$$
s_{u, u+1, t}=\frac{c_{i n v, u}-c_{i n v, u+1}}{c_{v a r, u+1, t}-c_{v a r, u, t}}
$$

with $u \in\{1,2, \ldots, n-1\}$ and a finite time horizon $t \in[0 ; T]$. Graphically $s_{u, u+1, t}$ represents the intersection of the cost curves of technology $u$ and $u+1$ (cf. figure 2). Each technology $u$ is directly competing against the adjacent technologies $u-1$ and $u+1$. If the resources were not constrained and there was no emission target, the upper and lower bound of efficient operation would be defined via the fixed $\operatorname{costs} c_{i n v, u}$ and 'pure' operating costs $c_{o p, u}$ (which is similar to the optimality condition in classical peak load pricing models). If scarce resources lead to a differing scarcity rent $h_{u} \cdot \lambda_{u, t}$ between neigbouring technologies, the operating bounds of $u$ change over time. Similar considerations apply if $\mathrm{CO}_{2}$-targets are tight and $\mathrm{CO}_{2}$-cost $\lambda_{p, t}$ become $<0$. With rising $\mathrm{CO}_{2}$-cost, the cleaner technologies have a cost advantage proportional to the differences in the specific emissions (as can be seen in (10)). As displayed in (12), 'pure' operating costs must be replaced by variable costs including also a scarcity rent and $\mathrm{CO}_{2}$-cost.

Under these circumstances a time-dependent covariable may obviously lead to an alteration of the efficient portfolio over time.

\subsection{Conditions for an efficient portfolio}

Releasing the prerequisite that all technologies are part of the efficiency border, it is possible that either some technologies are entirely dominated ${ }^{7}$ by a combination of the other ones or that a technology $u$ is not cost-minimal in the relevant operating time range $[0 ; S]$ (Refer to figure 3 for illustration). Then, an efficient portfolio $A^{*}$ may consist of any possible combination of technologies while the technology with lowest fixed cost $(u=n)$ is always part of it. ${ }^{8}$ For $n$ technologies $2^{n-1}$ possible permutations of technologies being part of the efficient portfolio are then possible.

Now, we show how to derive the technologies part of the efficient portfolio and hence the shape of the efficiency border if the cost curve of each technology is known. $s_{u, v}$ now describes any intersection of two arbitrary cost curves $u$ and $v$ with $u<v$. We maintain the assumption that all

\footnotetext{
${ }^{7}$ In the context of this paper, a technology that dominates another one in terms of cost shows lower cost.

${ }^{8}$ Accordingly to $\overrightarrow{s *}, A^{*}$ with $\left(A^{*} \subseteq A\right)$ marks technologies that are part of the efficiency border.
} 


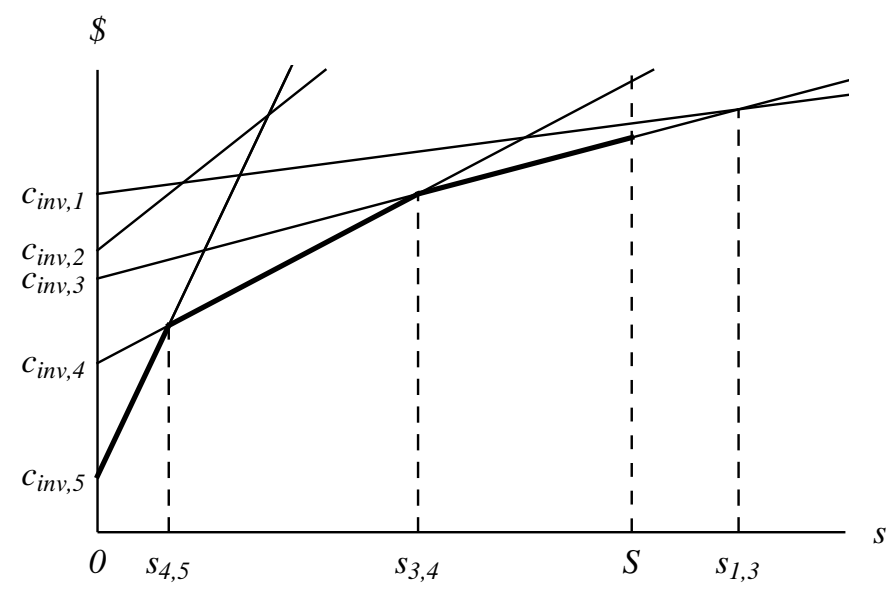

Figure 3: Formation of the efficiency border (fat line in graph) in a 5-technology-case. Technology 2 is dominated by a combination of other technologies, technology 1 is not cost-minimal during the relevant operating time interval $[0 ; S]$.

technologies are arranged in decreasing order of fixed costs, for variable costs no specific ordering is assumed in the following.

Proposition 1. Consider a technology $u$ in a set of technologies $A=\{1,2, \ldots, n\}$ strictly organized in decreasing order of fixed cost $c_{i n v, u}$. For technology $u \in A \backslash\{n\}$ to be part of the efficient portfolio it is sufficient that

$$
\begin{array}{r}
\forall w>u, 0<s_{u, w}<S \text { and } \\
\forall v<u,\left\{s_{v, u}<0 \vee \forall w>u, s_{u, w}<s_{v, u}\right\} .
\end{array}
$$

The technology $n$ with lowest fixed costs is part of the efficient portfolio in any case.

Proof. See B.1.

(14) describes the necessary condition that a technology $w$ with lower fixed costs $(w>u)$ has to have sufficiently high variable costs, so that the cost curve crosses the one of technology $u$ within the time range $[0 ; S]$. Otherwise technology $w$ will dominate technology $u$ over the entire time range.

The second condition ensures that a combination of multiple technologies does not disqualify technology $u$. Because the technologies are strictly ordered by decreasing fixed costs, a technology $v$ with higher investment cost than $u$ and with a cost curve crossing the one of $u$ before the lowcapital technology $w$ must be also subsequently cheaper than $u$ before $u$ would be cheaper than $w$. Hence $u$ would not be part of the efficient portfolio since it is first dominated by $w$ and then by $v$.

Corollary 1. Consider a technology $u \in A$ meeting Proposition 1. The efficient operating time 
of technology $u \in A \backslash\{n\}$ is then limited by

$$
\begin{array}{r}
\text { a lower bound } s_{u}^{\min }=\max _{w}\left(s_{u, w}\right), w>u \text { and } \\
\text { an upper bound } s_{u}^{\max }=\min \left\{\min _{v}\left(s_{v, u}\right) ; S\right\}, v<u .
\end{array}
$$

The lower bound of technology $n$ equals 0 and the upper bound is as described above.

Proof. See B.1.

If a technology $u$ is cost-minimal within a non-zero operating time range, this range is either limited by the maximum operating hours $S$ (zero) if the technology has lowest (highest) variable cost or by other technologies. If this is the case, all technologies with higher fixed cost, crossing the cost curve of $u$ have lower cost from the operating point of time on of the intersection. The minimum intersection consequently specifies the adjacent technology, respectively the upper operating time bound. For the lower bound similar conditions apply.

\subsection{Optimal solution for a general n-technology case}

In the following we further develop the specific optimization problem (5) building on the findings from the previous section. We extend it to a more general optimization problem factoring in the possibility that technologies might be dominated by others for e.g., a certain time period, but are part of the efficient portfolio otherwise. For this reason we first calculate a set of all possible solutions $\vec{s}$, than develop $\overrightarrow{s *}$, meaning the set of all $s_{v, u}$ that describe the efficiency border. From here installed capacities and resource use can be derived straightforwardly.

In section 3.1 we make the implicit assumption that strictly $s_{u, u-1}<s_{u-1, u-2}<\ldots<s_{2,1}<S$ as a necessary condition for (4) and (3) to hold true. When this requirement is released, we might experience an efficient portfolio $A^{*}$ consisting of only a subset of technologies as described in section 3.2.

So, consider a sequence of time intervals $\left[t_{i} ; t_{i+1}\right)$ denoted by $i \in 1,2, \ldots, I$ with $t_{1}=0$ and $t_{I+1}=\infty$. We assume that within each time interval i the subset of technologies $W_{i}$ does not change. The optimization problem within each time range can then be defined in a very similar way compared to the specific one in (5). We just replace the pool of available technologies in (3) and (4) by the pool of efficient technologies in the respective time interval:

$$
\begin{array}{r}
\max _{\substack{s_{u^{\prime}}^{\min }, s_{u^{\prime}}^{\max } u^{\prime} \in W_{i} \\
t_{i}}} \int_{t_{i}}^{t_{i+1}}\left[-\sum_{u^{\prime} \in W_{i}} c_{i n v, u^{\prime}} \cdot K_{u^{\prime}, t}\left(s_{u^{\prime}}^{\min }, s_{u^{\prime}}^{\max }\right)\right. \\
\left.-\sum_{u^{\prime} \in W_{i}} c_{o p, u^{\prime}} \cdot Q_{u^{\prime}, t}\left(s_{u^{\prime}}^{\min }, s_{u^{\prime}}^{\max }\right)\right] e^{-\rho t} d t .
\end{array}
$$

Subject to (1) and (2) where (3) and (4) are adjusted similar to (16), with $s_{u^{\prime}}^{\min }, s_{u^{\prime}}^{\max } \in \mathbb{R}^{+}$, 
optimality conditions are equivalently to (10)-(13) defined by

$$
\begin{aligned}
s_{u^{\prime}, v^{\prime}, t} & =\frac{c_{i n v, u^{\prime}}-c_{i n v, v^{\prime}}}{\left(c_{o p, v^{\prime}}-c_{o p, u^{\prime}}\right)+\left(h_{v^{\prime}} \cdot \lambda_{v^{\prime}, t}-h_{u^{\prime}} \cdot \lambda_{u^{\prime}, t}\right)-\lambda_{p, t}\left(\zeta_{v^{\prime}, t}-\zeta_{u^{\prime}, t}\right)} \\
& =\frac{c_{i n v, u^{\prime}}-c_{i n v, v^{\prime}}}{c_{v a r, v^{\prime}, t}-c_{v a r, u^{\prime}, t}}
\end{aligned}
$$

for all $u^{\prime}<v^{\prime}$ with $u^{\prime}, v^{\prime} \in W_{i} \subseteq A$ and $t \in\left[t_{i} ; t_{i+1}\right)$. Time-range specific parameters do not occur in the optimality conditions. Hence, $\vec{s}$ describing the intersections of all available technologies $A$ does not depend on the subset $W_{i}$ or the time interval $i .^{9}$ So, at time $t$ when actual cost parameters are given, $A_{t}^{*}$ is a subset of $A$ after applying Proposition $1, \vec{s}_{t} *$ derives respectively. Since we have extended the time horizon to infinity, the transversality conditions are defined as follows:

$$
\begin{array}{r}
\lim _{t \rightarrow+\infty} e^{-\rho t} \cdot \lambda_{u, t} \cdot X_{u, t}=0 \\
\lim _{t \rightarrow+\infty} e^{-\rho t} \cdot \lambda_{p, t} \cdot Z_{t}=0
\end{array}
$$

Summing, we advance the program (5) by (16) and propose the following:

Proposition 2. There exists an optimal solution to the program

$$
\begin{array}{r}
\max _{s_{u, v, t}, s_{w, u, t} u, v, w \in A} \int_{0}^{T}\left[-\sum_{u=1}^{n} c_{i n v, u} \cdot K_{u, t}\left(s_{w, u, t}, s_{u, v, t}\right)\right. \\
\left.-\sum_{u=1}^{n} c_{o p, u} \cdot Q_{u, t}\left(s_{w, u, t}, s_{u, v, t}\right)\right] e^{-\rho j} d t
\end{array}
$$

with (1)-(4) if there exists one non-polluting sustainable backstop technology with lowest operating costs.

Proof. See B.2.

\section{Application of the model with a polluting and a clean tech- nology}

In the following, we consider a two-technology case including a renewable technology $u=1$ and a fossil technology indexed by $u=3$. Later on we will extend the case to include also a $\mathrm{CO}_{2}$ abatement technology. The renewable is characterized by highest fixed and variable costs equal zero. ${ }^{10}$ Without loss of generality technology $u=3$ should burn gas and have relatively low fixed and operating costs $>0$. It should be polluting $\left(\zeta_{3}>0\right)$ and its productivity is described via a time-stable heat-rate $h_{3}>1$. We further assume the renewable technology not to be part of the

\footnotetext{
${ }^{9}$ Precisely, it depends in the way that the values will change over time, but its values are always defined by (18)

${ }^{10}$ The in the following derived solutions hold true also for operating costs $c_{o p, 1}>0$ (but still lowest) if we decrease the operating costs of the fossil technology by its difference to the actual operating costs of the renewable.
} 
efficient portfolio if no fossil scarcity or emission targets exist, i.e.

$$
s_{1,3}=\frac{c_{i n v, 1}-c_{i n v, 3}}{c_{o p, 3}} \geq S .
$$

The control variable

$$
s_{1,3, t}=\frac{c_{i n v, 1}-c_{i n v, 3}}{\left(c_{o p, 3}-c_{o p, 1}\right)+h_{3} \cdot \lambda_{3, t}-\zeta_{3} \cdot \lambda_{p, t}}
$$

describes the relevant control paths if technology 1 is part of the efficient portfolio.

\subsection{A small initial reserve making $\mathrm{CO}_{2}$-restrictions obsolete}

Let us first assume that $X_{3}^{0}$ is small enough, so an emission ceiling would never be binding. This results in a pure Hotelling price path with $\lambda_{3, t}=\lambda_{3,0} \cdot e^{\rho t}$ (compare with (8)) from the very beginning $\left(\lambda_{p, t} \equiv 0\right)$. The time derivative $\frac{\partial s_{1,3, t}}{\partial t}=-\frac{\left(c_{i n v, 1}-c_{i n v, 3}\right) \rho \cdot h_{3} \cdot \lambda_{3,0} \cdot e^{p \cdot t}}{\left(c_{o p, 3}-h_{3} \cdot \lambda_{3,0} \cdot e^{\rho \cdot t}\right)^{2}}$ is strictly negative and leads to a continuously decreasing $s_{1,3, t}$ which converges to 0 when time approaches infinity. Because of (20), on a cost minimum time path the initial resource stock must be consumed completely. So, by $\int_{0}^{\infty} x_{3, t} d t=X_{3}^{0}$ there's a well-defined price path $\lambda_{3,0}\left(X_{3}^{0}\right)$. Refer to Appendix B.4 for details.

The larger the initial stock, the larger $s_{1,3,0}$ and respectively the initial share of gas within the energy mix. $X_{3}^{R}$ should define the critical reserve which leads to an $s_{1,3,0}$ exactly equal to $\mathrm{S}$. Then, for all $X_{3}^{0}<X_{3}^{R}, s_{1,3,0}<S$. If the initial reserve $X_{3}^{0}$ is larger than $X_{3}^{R}$, gas is exclusively used in the beginning and the renewable resource complements the energy mix as soon as $X_{3, t}$ has decreased to a critical reserve. We label the time when the renewable technology enters the portfolio with $\theta_{R} \cdot{ }^{11}$ At a fixed initial reserve, the more competitive the renewable technology is, the earlier it complements the energy mix and hence the longer lasts the scarce resource. Figure 4 illustrates the control path as described and the corresponding cost curves of the renewable and gas at $t_{1}$ when the renewable is not yet part of the portfolio and at $t_{2}$ where we see a twotechnology mix. Compare the right-hand side with figure 2 which displays similar curves with flipped axes. Contrarily to the pure Hotelling model without emission constraint, there is not a one-time flip from the scarce resource to the backstop technology. Rather we see a coexistence of both technologies.

\subsection{A large initial reserve making $\mathrm{CO}_{2}$-restrictions relevant}

If the initial reserve is large enough, the optimal consumption path depends on a politically defined carbon ceiling. We first develop the condition which makes the carbon ceiling relevant. Subsequently, we describe potential extraction paths.

$\lambda_{3,0}$ decreases with increasing $X_{3}^{0}$. The continuously over time decreasing $s_{1,3, t}$ leads to a decreasing use of gas. ${ }^{12}$ Hence, if the initial carbon emission is less or equal to the natural dilution at $t=0$, the carbon content in the atmosphere continuously decreases. If the carbon emission exceeds the natural dilution in the beginning, the carbon content $Z_{t}$ continuously increases, peaks

\footnotetext{
${ }^{11}$ It is defined via the time $t>0$ when $s_{1,3, t}$ equals $S$.

${ }^{12} \mathrm{An}$ initial phase where the renewable energy is not yet part of the efficient portfolio would lead to a constant use of gas for $t \leq \theta_{R}$.
} 

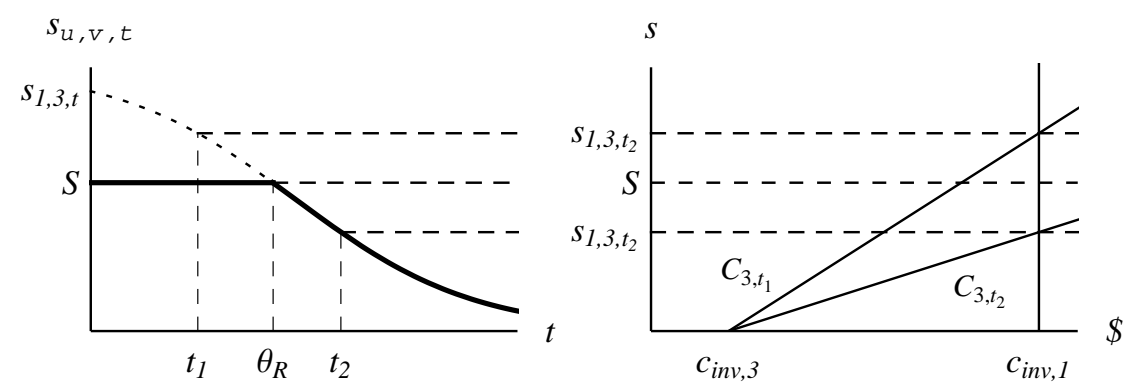

Figure 4: Portfolio time-path with a scarce resource $(\mathrm{u}=3)$ and a backstop technology $(\mathrm{u}=1)$ and corresponding cost curves (see right hand side) at two distinct points of time.

at a certain time $t$ and decreases afterwards. The larger $X_{3}^{0}$, the higher the maximum. Hence, there exists a $X_{3}^{H}$ which leads to a carbon content peaking at exactly $\bar{Z}$. If the initial reserve is lower than $X_{3}^{H}$, the carbon ceiling is obsolete, if it is higher $\lambda_{p, 0}<0$ within a non-zero initial time period (cf. Chakravorty et al. (2008)).

Under the assumptions we make, the efficient path should imply an initial phase of exclusive use of the polluting resource if the ceiling is relevant. We then see a three-phase extraction sequence as shown in figure 5: In the first phase, the demand is exclusively met via the polluting resource, $Z_{j}$ continuously increases and the renewable resource enters the portfolio at $\theta_{R}$. The variable costs of gas rise with

$$
c_{v a r, 3, t}\left(\lambda_{3,0}, \lambda_{p, 0}, t\right)=c_{o p, 3}+h_{3} \cdot \lambda_{3,0} \cdot e^{\rho \cdot t}-\zeta_{3} \cdot \lambda_{p, 0} \cdot e^{(\rho+\alpha) \cdot t} .
$$

$s_{1,3, t}^{c}=\frac{c_{i n v, 1}-c_{i n v, 3}}{c_{v a r, 3, t}}$, where the superscript stands for relevant ceiling $\left(\equiv \lambda_{p, t}<0\right)$, decreases respectively. $\dot{Z}_{t}>0$, but decreasing over time until $Z_{t}$ reaches the ceiling at $\theta_{C}$ where the subscript stands for ceiling. During the second phase, carbon emissions through resource use and natural dilutions are balanced, $Z_{t}=\bar{Z}$ and $\bar{s}_{1,3}$, where the bar stands for constant and balanced emissions, are consequently constant. This ceiling phase is defined by $\zeta_{3} \cdot \int_{0}^{\bar{s}_{1,3}} D(s)-\bar{s}_{1,3} d s=\alpha \cdot \bar{Z}$. It implies, at a constantly increasing scarcity rent, decreasing $\mathrm{CO}_{2}$-cost. They phase out as soon as the scarcity rent is high enough to limit emissions to $\dot{Z}_{t}<\alpha \cdot \bar{Z}$ at $\theta_{H}=\rho^{-1} \ln \left(-\frac{c_{i n v, 3}-c_{i n v, 1}+c_{o p, 3} \cdot \bar{s}_{1,3}}{h_{3} \cdot \lambda_{3,0} \cdot \bar{s}_{1,3}}\right)$ when the third phase begins. Gas is then used until infinity at a constantly decreasing share. See B.4 for a detailed characterization of the control paths.

Proposition 3. Consider initial endowments with fossil resources large enough to make a $\mathrm{CO}_{2^{-}}$ ceiling relevant. A consequential initially emission constrained control path always results in a ceiling phase before a pure Hotelling path begins. This ceiling phase, where the $\mathrm{CO}_{2}$-content in the atmosphere stays at the maximum level, is characterized by a net-emission equal zero while natural dilution is balanced by emissions through fossil combustion.

Proof. See B.3.

\subsection{Introduction of an option for carbon capturing}

Carbon capture and storage (CCS) provides a technology that allows the use of fossil fuels for electricity production while a large share of the $\mathrm{CO}_{2}$ produced is held back. This comes with 


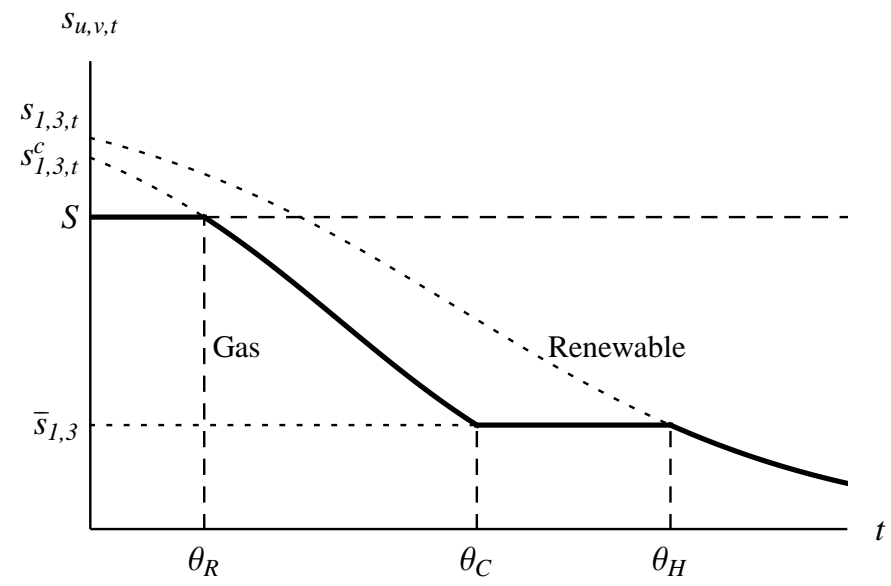

Figure 5: Portfolio time paths with one initial stock available and $\mathrm{CO}_{2}$-restrictions (Note: $s_{1,3, t}^{c}=$ $f\left(\lambda_{3,0}, \lambda_{p, 0}, t\right)$ and $s_{1,3, t}=f\left(\lambda_{3,0}, t\right)$ with $\lambda_{1,3, t}=\lambda_{3,0} \cdot e^{\rho t}$ and $\left.\lambda_{p, t}=\lambda_{p, 0} \cdot e^{(\rho+\alpha) t}\right)$

increased costs as well as an efficiency malus compared to the non-CCS counterparts. Hence, without tight emission targets, the CCS technology can never be economical.

We introduce gas CCS as an additional technology $u=2$ with highest operating costs $\left(c_{o p, 2}>\right.$ $\left.c_{o p, 3}\right)$, medium investment costs $\left(c_{i n v, 3}<c_{i n v, 2}<c_{i n v, 1}\right)$ and lowest efficiency $\left(h_{2}>h_{3}\right)$, as generally proposed in literature (e.g., İşlegen and Reichelstein (2011)). ${ }^{13}$ Consider a potential time path where a CCS technology becomes part of the efficient portfolio and refer to figure 6 for illustration. The path shows the three-phase appearance which characterizes every path with relevant $\mathrm{CO}_{2}$-targets. In the beginning, gas is the only technology used, until at $\theta_{R}$ the renewable complements the portfolio and its share continuously increases. The variable costs of non-CCS gas rise quicker than those of gas CCS which does not show any $\mathrm{CO}_{2}$-costs. ${ }^{14}$ As soon as $\zeta_{3} \cdot \lambda_{p, t}>c_{o p, 2}-c_{o p, 3}+\lambda_{p, t} \cdot\left(h_{2}-h_{3}\right)$, gas CCS has lowest variable costs. It becomes distinct by an intersection between the cost curves of technology $u=2$ and $u=3$ (appearance of $s_{2,3, t}^{c}$ in the positive range). ${ }^{15}$ But still, due to the higher investment costs in comparison to non-CCS gas, gas CCS is dominated by the combination of the remaining two technologies. At $t=\theta_{C C S_{1}}$, the increased $\mathrm{CO}_{2}$-costs have also compensated the investment malus and gas CCS enters the portfolio. The following equotation reflects this situation when $s_{1,3}^{c}$ equals $s_{1,2}^{c}$ :

$$
\frac{c_{i n v, 1}-c_{i n v, 2}}{c_{i n v, 1}-c_{i n v, 3}}=\frac{c_{o p, 2}+h_{2} \cdot \lambda_{3, t}}{c_{o p, 3}+h_{3} \cdot \lambda_{3, t}-\zeta_{3} \cdot \lambda_{p, t}}
$$

We see the ratio of cost advantage compared to the renewable in terms of fixed costs on the left-hand side compared to the ratio of variable costs of gas and gas CCS on the right-hand side.

Now, the share of non-CCS gas decreases to $\bar{s}_{2,3}$ at $\theta_{C}$ when $Z_{t}=\bar{Z}$. The following ceiling phase is characterized by a constant share of non-CCS gas and a continuously decreasing share of gas CCS until it leaves the portfolio at $t=\theta_{\mathrm{CCS}_{2}}$. Before a pure Hotelling path begins, a stable non-zero time path $t \in\left[\theta_{\mathrm{CCS}_{2}} ; \theta_{H}\right]$ with non-CCS gas and the renewable technology follows,

\footnotetext{
${ }^{13}$ Both technologies gas and gas CCS use the same resource and show the same scarcity rent $\lambda_{3, t}$.

${ }^{14}$ Compare $c_{v a r, 2, t}=c_{o p, 2}+h_{2} \cdot \lambda_{3,0} \cdot e^{\rho t}$ and $c_{3, v a r, t}=c_{o p, 3}+h_{3} \cdot \lambda_{3,0} \cdot e^{\rho t}-\zeta_{3} \cdot \lambda_{p, 0} \cdot e^{(\rho+\alpha) t}$

${ }^{15}$ Before, (18) is negative with $u=2$ and $v=3$ since the difference in operating costs is still positive and the difference in investment costs is negative.
} 
defined via $\bar{s}_{1,3}\left(=\bar{s}_{2,3}\right)$.

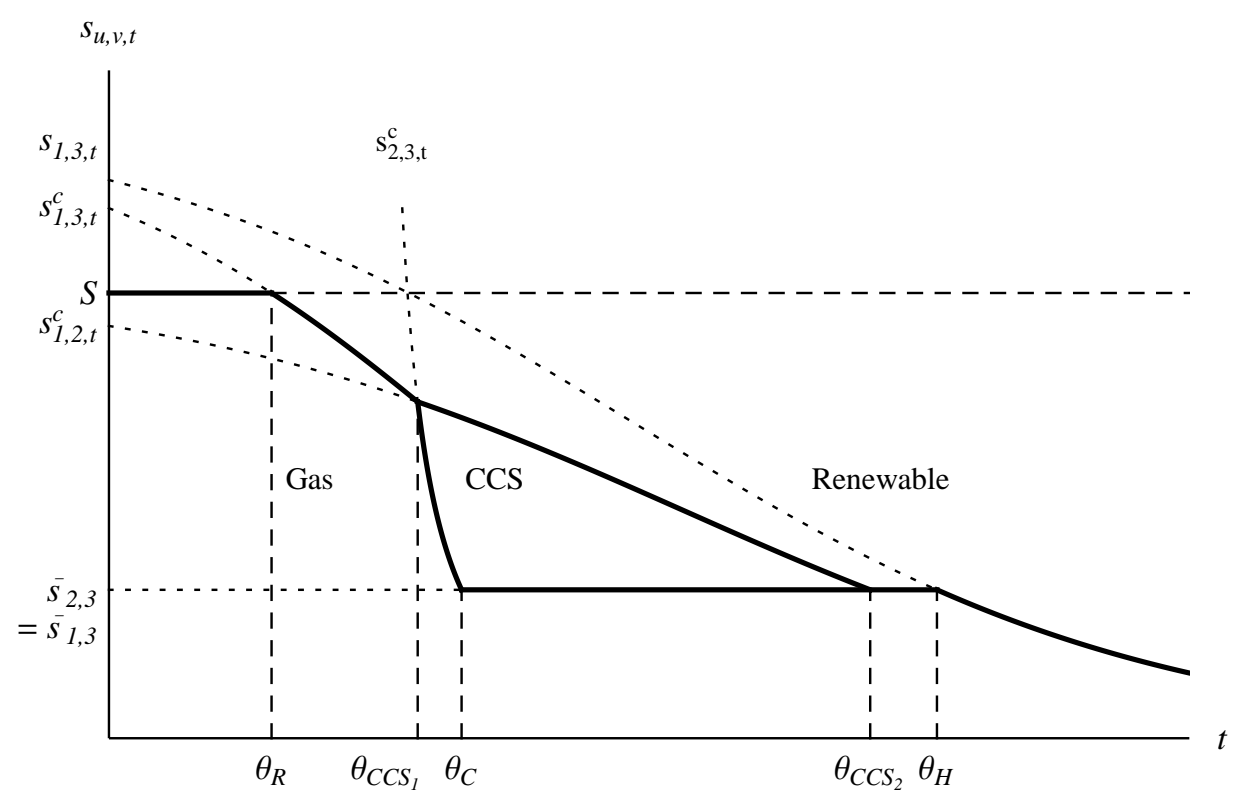

Figure 6: Portfolio time paths with one initial stock available, a CCS technology and $\mathrm{CO}_{2}{ }^{-}$ restrictions

We now describe the conditions that lead for CCS to be part of the efficient portfolio and specify its entry and exit point of time. Referring to Proposition 3, a non-zero time ceiling phase exists if emission targets are tight. This is a necessary condition for CCS to become part of the efficient portfolio. Hence, it can only be part of the portfolio as long as $\lambda_{p, t}<0$, so the constrained paths $s_{u, v, t}^{c}$ are relevant. According to Proposition 1, gas CCS is part of the efficient portfolio if $s_{1,3, t}^{c}<s_{1,2, t}^{c}$ and $s_{1,3, t}^{c}<S$. (21) describes the situation where $s_{1,3, t}^{c}=s_{1,2, t}^{c}$. And $s_{1,2, \theta_{C C S_{1}}}^{c}$ should specify the operating hours at this point of time. Then, if $s_{1,2, \theta_{C C S_{1}}}>\bar{s}_{1,3}\left(=\bar{s}_{2,3}\right)$, gas CCS will be part of the efficient portfolio for a non-zero time period. Otherwise it will not since $s_{1,2, t}^{c}$ is strictly larger than $s_{2,3, t}^{c}$ from that point of time on and, according to Proposition 1, technology 1 and 3 dominate gas CCS. ${ }^{16}$

Gas CCS will leave the portfolio as soon as $s_{1,2}^{c}$ equals $\bar{s}_{2,3}$. At this point of time the further increased scarcity rent and decreased $\mathrm{CO}_{2}$-costs make the CCS technology no further economical. This is strictly before a pure Hotelling path begins and results in a stable non-CCS gas-renewable mix until $\mathrm{CO}_{2}$-costs $\lambda_{p, t}$ have further decreased to zero at $\theta_{R}$. It is the case because $s_{1,2, t}^{c}$ (which equals $s_{1,2, t}$ since technology 2 is clean) is strictly lower than $s_{1,3, t}$ due to the higher costs and lower efficiency of CCS compared to normal gas. Hence, $s_{1,2, t}^{c}$ must cut $\bar{s}_{2,3}$, respectively $\bar{s}_{1,3}$ before $s_{1,3, t}$ does.

\subsection{Review on scarcity rents}

The response of economic sectors to reduction efforts has been widely discussed in the literature (e.g., in Eisenack et al. (2012)). In our assessment, a complete exploitation of scarce resources

\footnotetext{
${ }^{16}$ If $s_{1,2, \theta_{C C S_{1}}^{c}}>S$, CCS will enter the efficient portfolio before the renewable does, the entry point of time is then between $\theta_{C C S_{1}}$ and $\theta_{R}$. It would be defined by the time when $s_{2,3, t}^{c}$ equals $\mathrm{S}$.
} 
is necessary for a cost-minimum solution. We conclude that resource owners would likely oppose emission targets from an economic perspective, while CCS could decrease societal costs while upholding scarcity rents.

Consider a resource owner that maximizes profit in the way that he fully exploits resources at maximum scarcity rent. ${ }^{17}$ Further consider the initial resource stock as given and so is also the scarcity rent in the case described in section 4.1 (三 non-constrained path). We now introduce an effective carbon ceiling as shown in section 4.2 (三 constrained non-CCS path) and hold on to the scarcity rent. Hence, the Hotelling path $s_{1,3, t}$ is congruent with the one in the non-constrained case. But the initial constrained path $s_{1,3, t}^{c}$ and the ceiling path $\bar{s}_{1,3}$ are strictly below $s_{1,3, t}$ during their effective phase. So, the resource exploited at $\theta_{H}$ is less than on the non-constrained path. For $t>\theta_{H}$, the relevant control paths of both cases are identical and so is the consumption of gas during this time interval. Hence, to ensure a complete exploitation at infinity, the scarcity rent $\lambda_{3,0}$ must be strictly lower on the constrained path. So, considering the scarcity rent only, a resource owner would consequently oppose emission targets from an economic perspective. ${ }^{18}$ The CCS technology allows to retain a higher share of the scarce resource in the portfolio for $t<\theta_{H}$. Though, it was still lower than in the unconstrained one. So, CCS could help to soften scarcity rent losses to resource owners if emission targets are introduced.

\section{Conclusion}

This paper combines Hotelling's theory, that was for the first time extended to an emission constrained environment by Tahvonen (1997), with a peak load pricing approach to adequately describe electricity production systems with simultaneous resource and emission constraints. The maximum amount of $\mathrm{CO}_{2}$ in the atmosphere as well as the resource stocks are constrained. The demand is fixed and characterized by a load duration curve which is used to illustrate the relationship between generating capacity requirements and capacity utilization in electricity production. Our approach singles out from existing literature by focusing on the electricity production which allows us to take fixed costs and fluctuating demand into consideration which are essential to describe electricity markets. For the illustration of possible extraction paths, we use different cases which include a polluting technology which relies on a fossil fuel (e.g. gas) as input factor and is characterized by relatively low fixed, but high operating cost. A green, supply-independent, technology with high fixed and no variable costs complements the technology portfolio. Furthermore, an option for carbon capturing is introduced.

One general result is that in all cases gas is exploited completely at infinity. In the beginning it is used exclusively. Beyond this interval, the share of the renewable will continuously increase while the use of gas continuously decreases. When the initial stocks are high enough (or emission targets tight enough), a ceiling phase results. During this ceiling phase, the share of gas will remain stable. This phase will, if once reached, last relatively long. An also gas-based carbon capturing technology, which has higher investment and operating costs than its non-CCS counterpart, could complement the portfolio as an intermediate technology. It would then enter the portfolio before

\footnotetext{
${ }^{17}$ We still stick to the optimization approach as described in (19).

${ }^{18} \mathrm{~A}$ damage function that would also take economic impacts of climate change into account may revert this conclusion.
} 
the ceiling is reached and leave it before a final Hotelling path begins.

We confirm findings from related literature that renewables should complement the portfolio despite of higher unit cost. The same holds true for a carbon capturing technology. With the assumptions we make, CCS may complement the portfolio and then help to decrease societal cost of electricity production. Findings suggest that resource owners are likely to oppose emission targets since a target would always result in an decrease of scarcity rents. CCS would also help to limit losses of scarcity rents which may increase acceptance among resource owners.

Modeling the development over time of a portfolio consisting of a fossil and a renewable technology is a first step in including fixed costs into existing theory. An inclusion of an additional fossil resource with different cost and polluting characteristics could help to understand the potential paths and policy implications for multiple fossil resources available.

Furthermore, we add to classical peak load pricing literature a new approach to derive the efficient production portfolio at known fixed and variable costs using the intersection of multiple cost curves as a function of operating time. The shortcoming of this peak load pricing framework which does not accommodate for fluctuating energy feed-in of renewable technologies like solar or wind and assumes a fixed price-inelastic demand should be addressed in further research. 


\title{
A Symbols and model notation
}

\author{
Indices \\ $u, v, w \quad$ Generation technology, fuel type \\ $s \quad$ Share of total operating time per period \\ (e.g., similar to operating hours per year) \\ $t \quad$ Point of time in the continuous series of multiple analysis periods \\ $i \quad$ Time interval during which the composition of the efficient portfolio does not change
}

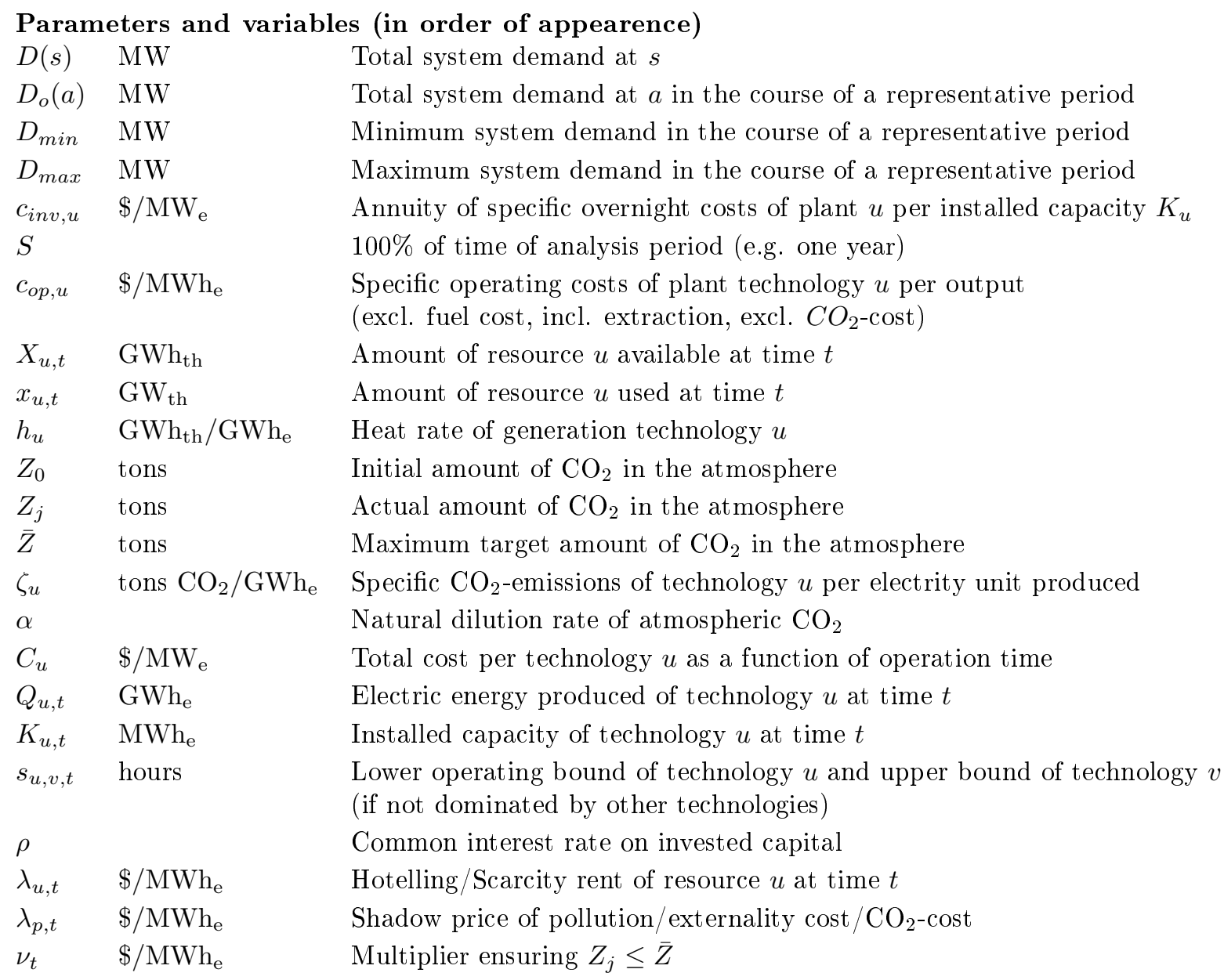




\section{Vectors and other}

A

$A_{t}^{*}$

$W_{i}$

$\overrightarrow{s_{t}} *$

$\overrightarrow{s_{t}}$

$\theta_{u, E}$

$\theta_{C}$

$\theta_{H}$

$X^{H} \quad \mathrm{GWh}_{\text {th }}$

$X^{R} \quad \mathrm{GWh}_{\mathrm{th}}$
Set of available technologies

Subset of technologies $A$ that are part of the efficient portfolio at $t$

Reduced set of technologies that would be part of the efficient portfolio in the time interval $i$ )

Vector describing relevant limits of operating time

between technologies $A_{t}^{*}$ that are part of the efficient portfolio at $t$

Vector describing every intersection between all

cost curves of technologies available $A$ at time $t$

Time when resource $u$ is exploited

Time when $Z_{t}$ reaches the ceiling

Time when a pure Hotelling path begins

Initial amount of coal for which an infinitesimally

higher initial endowment results in an initial non-Hotelling path

Initial amount of coal for which an infinitesimally

higher initial endowment results in an initial pure fossil path 


\section{B Proofs and mathematical appendix}

\section{B.1 Proof of Proposition 1 and Corollary 1}

Part a: u part of the efficient portfolio implies (14) and (15)

Consider two technologies $u, w \in A$ with $c_{i n v, u}>c_{i n v, w}$. The costs of both technologies may be written:

$$
C_{u}(s)=c_{i n v, u}+c_{v a r, u} \cdot s \quad C_{w}(s)=c_{i n v, w}+c_{v a r, w} \cdot s .
$$

The cost difference is then:

$$
C_{u}(s)-C_{w}(s)=c_{i n v, u}-c_{i n v, w}+\left(c_{v a r, u}-c_{v a r, w}\right) \cdot s .
$$

The cost difference is decreasing with increasing operating time $s$ if and only if

$$
c_{v a r, w}>c_{v a r, u}
$$

The operating time $s_{u, w}$, where both technologies have the same cost, equals

$$
s_{u, w}=\frac{c_{i n v, u}-c_{i n v, w}}{c_{v a r, w}-c_{v a r, u}} .
$$

A necessary condition for technology $u$ to be cost efficient is hence

$$
s_{u, w}>0
$$

This corresponds to the first inequality in equation (14). Only for $s>s_{u, w}$, the cost of technology $u$ is then smaller than the cost of $w$. Since the maximum possible operating time is $S$, a further inequality has to be satisfied:

$$
s_{u, w}<S
$$

This is the second inequality in equation (14).

Additionally the technology $u$ may not be undercut in costs by technologies $v$ with higher investment $\operatorname{costs} c_{i n v, v}>c_{i n v, u}$, at least not before it had its part on the efficient cost curve. As before

$$
\begin{gathered}
s_{v, u}=\frac{c_{i n v, v}-c_{i n v, u}}{c_{v a r, u}-c_{v a r, v}} . \\
\text { If } s_{v, u}<0,
\end{gathered}
$$

then the cost difference $C_{v}(s)-C_{u}(s)$ is positive and increasing for positive $s$, thus this is a sufficient condition for no cost undercut by technology $v$. Otherwise the cost of technology $v$ is below the one of $u$ beyond the point $s_{v, u}$. If for any combination of $u, v$ and $w s_{v, u}$ was smaller than $s_{u, w}$ then $u$ would be dominated by $w$ in cost terms until $s_{u, w}$ and beyond $s_{u, w} u$ would 
already be dominated by $v$ given that $s_{v, u}<s_{u, w}$. Hence a necessary condition under $s_{v, u}>0$ is:

$$
\forall v<u, \forall w>u, s_{u, w}<s_{v, u}
$$

This is the second part of equation (15).

\section{Part b: (14) and (15) imply u part of the efficient portfolio}

Given that we are looking at a finite set of technologies, (14) implies that there is a minimum operation time $s_{u}^{\min }$ from which on technology $u$ undercuts the costs of all technologies $w$ with lower investment costs:

$$
s_{u}^{\min }=\max _{w w>0} s_{u, w}
$$

Equation (14) moreover implies:

$$
s_{u}^{\text {min }}<S
$$

Similarly, there is a maximum operation time $s_{u}^{\max 0}$ with

$$
s_{u}^{\max 0}=\min _{v v<u s_{v, u}>0} s_{v, u} .
$$

Below that operation time, technology $u$ has lower costs than all technologies $v$ with higher investment costs. Equation (15) then implies

$$
s_{u}^{\min }<s_{u}^{\max 0}
$$

However, it is not clear a priori whether $s_{u}^{\max 0}$ is smaller or larger than $S$. Since operating times larger than $S$ are not meaningful we define:

$$
s_{u}^{\max }=\min \left\{s_{u}^{\max 0}, S\right\}
$$

Consequently technology $u$ is cost efficient in the interval $\left[s_{u}^{\min }, s_{u}^{\max }\right]$ and thus part of the efficient set.

\section{B.2 Existence of an optimal solution}

Following Chakravorty and Krulce (1994) and Farzin (1992), we use Seierstad and Sydsæter (1987, Theorem 15, p. 237) to prove the existence of an optimal solution to the program described. The backstop technology is assumed to have lowest operating costs. Consequently, it dominates all other technologies with higher fixed costs for $t \geq 0 .{ }^{19}$ So, we assume the backstop to be technology $u=1$ with highest fixed costs. We define the integrand of $(19)$ as $f_{0}\left(s_{u, v, t}, t\right),(1)$ as $f_{u}\left(s_{u, v, t}, t\right)$ with $x_{u, t}=h_{u} \cdot Q_{u, t}\left(s_{w, u, t}^{*}, s_{u, v, t}^{*}\right)$ for $u=\{1,2, \ldots, n\}^{20}$ and (2) as $f_{n+1}\left(s_{u, v, t}, t\right)$ with identical definitions for $x_{u, t}$. Similar to the main paper we specify the set of all intersections of different

\footnotetext{
${ }^{19}$ Both the Hotelling rent and the external cost of pollution equal zero for a backstop technology.

${ }^{20}$ Recall that backstop technology $u=1$ does not rely on depletive input factors, so $f_{1}\left(s_{u, v, t}, t\right)$ does not exist and will not be considered further.
} 
cost curves $s_{u, v, t}$ with $\vec{s}_{u, v, t} ; \vec{s}_{u, v, t}^{*}$ should specify the relevant control paths where $s_{u, v, t}^{*}$ limits the efficient operating time of two technologies $u$ and $v$ that are part of the efficient portfolio at time $t$.

Condition 1: The paths of the control variables specified in $\vec{s}_{u, v, t}^{*}$ are piecewise continuous on $[0 ; \infty)$.

Proof: Each $s_{u, v, t}$ may qualify as a relevant control variable.

$s_{u, v, t}=\frac{c_{i n v, u}-c_{i n v, v}}{\left(c_{o p, v}-c_{o p, u}\right)+\left(h_{v} \cdot \lambda_{v, t}-h_{u} \cdot \lambda_{u, t}\right)-\lambda_{p, t}\left(\zeta_{v, t}-\zeta_{u, t}\right)}$, where the dynamics of $\lambda_{u, t}$ and $\lambda_{v, t}$ are specified in (7)-(9). Inspection of the curves specified in $\vec{s}_{u, v, t}$ reveals that they are all piecewise continuous. The same holds consequently true for $\vec{s}_{u, v, t}^{*}$ since $A_{t}^{*} \subseteq A$.

Condition 2: The control variables specified in $\vec{s}_{u, v, t}^{*}$ are bounded on $[0 ; \infty)$.

Proof: According to Proposition 1 and since the demand $D(s)$ is defined only for $s \in[0 ; S]$, a $s_{u, v, t}^{*} \notin[0 ; S]$ would disqualify itself from being a relevant control variable. Consequently all relevant control variables $\vec{s}_{u, v, t}^{*}$ are bounded.

Condition 3: $f_{u}\left(s_{u, v, t}, t\right)$ is continuous for all $u=\{0, \ldots, n+1\}$.

Proof: Since $D(s)$ is assumed to be continuously decreasing in $s, Q_{u, t}\left(s_{w, u, t}^{*}, s_{u, v, t}^{*}\right)$ and $K_{u, t}\left(s_{w, u, t}^{*}, s_{u, v, t}^{*}\right)$ defined by (3) and (4) must then also be continuous if all $s_{u, v, t}^{*}$ are continuous. $s_{u, v, t}$ shows only discontinuities where it changes algebraic signs. Suspection of the function reveals that this takes place when it switches from $-\infty$ to $\infty$ or vice versa. According to Proposition 1 and Condition $2, s_{u, v, t}$ is not a relevant control variable where it shows discontinuities. Hence, all $\vec{s}_{u, v, t}^{*}$ are continuous and so are $Q_{u, t}\left(s_{w, u, t}^{*}, s_{u, v, t}^{*}\right)$ and $K_{u, t}\left(s_{w, u, t}^{*}, s_{u, v, t}^{*}\right)$. Consequently $f_{u}\left(s_{u, v, t}, t\right)$ is also continuous for all $u=2, \ldots, n$. The same holds true for $f_{0}\left(s_{u, v, t}, t\right)$ since it is then a sum of continuous functions. $f_{n+1}\left(s_{u, v, t}, t\right)$ must then also be continuous for the same reason if $\alpha \cdot Z_{t}$ is continuous. If we assume $f_{n+1}\left(s_{u, v, t}, t\right)$ to be continuous its antiderivative $Z_{t}$ is also continuous. Consequently, $f_{n+1}\left(s_{u, v, t}, t\right)$ is continuous.

Condition 4: There exists a piecewise continuous function $\phi_{0}(t) \geq 0$ with $\int_{0}^{\infty} \phi_{0}(t) d t<\infty$ such that $f_{0}\left(s_{u, v, t}, t\right) \leq \phi_{0}(t)$ for any admissible path $\vec{s}_{u, v, t}^{*}$ with $t \geq 0$.

Proof: Since for all $u \in\{1,2, \cdots, n\} c_{i n v, u}, c_{o p, u}, K_{u, t}$ and $Q_{u, t}$ are non-negative $f_{0}\left(s_{u, v, t}, t\right) \leq 0$. So, define $\phi_{0}(t)=0$ for all $t \geq 0$ to obtain the result.

Condition 5: There exists a piecewise continuous functions $\phi_{u}(t) \geq 0$ with $\int_{0}^{\infty} \phi_{u}(t) d t<\infty$ such that $\left|f_{u}\left(s_{u, v, t}, t\right)\right| \leq \phi_{u}(t)$ for any admissible path specified in $\vec{s}_{u, v, t}^{*}$ for all $u=2,3, \ldots, n$ and $t \geq 0$.

Proof: $\left|f_{u}\left(s_{u, v, t}, t\right)\right|=\left|-h_{u} \cdot Q_{u, t}\right|=\left|-h_{u} \cdot x_{u, t}\right|$ with $\int_{0}^{\infty} x_{u, t} d t \leq X_{u}^{0}$ for all $u=2,3, \ldots, n$. Further consider a case where $s_{1, u, t}=S$ and $s_{1, v, t}=0$ for all $v=\{2,3, \ldots, n\} / u$ such that $\left|f_{u}\left(s_{u, v, t}, t\right)\right|$ forms its maximum possible value which equals $\left|-h_{u} \cdot Q_{u, t}(0, S)\right|=\left|-h_{u} \cdot \int_{0}^{S} D(s) d s\right|<\infty$. So for any admissible control path $\left|f_{u}\left(s_{u, v, t}, t\right)\right|$ is upward bound and its integral $<\infty$ and consequently there exists a $\phi_{u}(t)=\left|f_{u}\left(s_{u, v, t}, t\right)\right|$ with $\phi_{u}(t) \geq 0$ and $\int_{0}^{\infty} \phi_{u}(t) d t<\infty$ for all $u=2,3, \ldots, n$ and $t \geq 0$. 
Condition 6: There exists a piecewise continuous functions $\phi_{n+1}(t) \geq 0$ with $\int_{0}^{\infty} \phi_{n+1}(t) d t<\infty$ such that $\left|f_{n+1}\left(s_{u, v, t}, t\right)\right| \leq \phi_{n+1}(t)$ for any admissible path specified in $s_{u, v, t}^{*}$ for all $t \geq 0$.

Proof: Inspection of the function reveals that $-\alpha \cdot \bar{Z} \leq f_{n+1}\left(s_{u, v, t}, t\right) \leq \zeta_{w} \cdot \int_{0}^{S} D(s) d s$ with $\zeta_{w} \geq \zeta_{u}$ for all $u=1,2, \cdots, n$. So, $\left|f_{n+1}\left(s_{u, v, t}, t\right)\right| \leq \max \left\{|-\alpha \cdot \bar{Z}|,\left|\zeta_{w} \cdot \int_{0}^{S} D(s) d s\right|\right\}=J<\infty$ for $t \geq 0$. Furthermore, due to the constrained fossil polluting resources (and the continuity of $Q_{u, t}$ ), the share of all fossil resources must eventually continuously decrease to zero (c.f. also the final Hotelling path in Chakravorty et al. (2008)). Define $t_{1}$ such that $-\alpha \cdot Z_{t}>\sum_{u=1}^{n} \zeta_{u} \cdot Q_{u, t}$ for all $t>t_{1}$. For this eventual phase $\left|f_{n+1}\left(s_{u, v, t}, t\right)\right|=\left|\dot{Z}_{t}\right|=\left|-\alpha \cdot Z_{t}+\sum_{u=1}^{n} \zeta_{u} \cdot Q_{u, t}\right|<\left|-\alpha \cdot Z_{t}\right|$. So, there must exist a positive $c<\infty$ such that $\left|f_{n+1}\left(s_{u, v, t}, t\right)\right| \leq\left|-c \cdot \alpha e^{-\alpha t}\right|$ for all $t \in\left(t_{1} ; \infty\right)$. Define $\phi_{n+1}(t)=J$ for $t \in\left[0 ; t_{1}\right]$ and $\phi_{n+1}(t)=\left|-c \cdot \alpha e^{-\alpha t}\right|$ for $t \in\left(t_{1} ; \infty\right)$ to obtain the result. So, $\phi_{n+1}(t) \geq 0$ with $\int_{0}^{\infty} \phi_{n+1}(t) d t=t_{1} \cdot J+\int_{t_{1}}^{\infty}\left|-c \cdot \alpha e^{-\alpha t}\right|<\infty$ and $\left|f_{n+1}\left(s_{u, v, t}, t\right)\right| \leq \phi_{n+1}(t)$ for any admissible path specified in $\vec{s}_{u, v, t}^{*}$ for all $t \geq 0$.

Condition 7: There exist piecewise continuous, non-negative functions $a(t)$ and $b(t)$ such that $\left|f_{u}\left(s_{u, v, t}, t\right)\right| \leq a(t) \cdot|x|+b(t)$ for all $u=2, \ldots, n+1$ and $t \geq 0$.

Proof: In Condition 5 and 6 we show that $\left|f_{u}\left(s_{u, v, t}, t\right)\right|$ is bounded above by $\int_{0}^{S} h_{u} D(s) d s<\infty$ for all $u=2, \cdots, n$ and by $\max \left\{|-\alpha \cdot \bar{Z}|,\left|\zeta_{w} \cdot \int_{0}^{S} D(s) d s\right|\right\}<\infty$ for $u=n+1$. Let $b(t)$ be this bound and $a(t)=0$ to obtain the result.

Condition 8: The set $N\left(s_{u, v, t}, t\right)=\left\{\left(f_{0}\left(s_{u, v, t}, t\right)+\gamma, f_{2}\left(s_{u, v, t}, t\right), \ldots, f_{n+1}\left(s_{u, v, t}\right)\right): s_{u, v, t} \in\right.$ $[0 ; S], \gamma \leq 0\}$ is convex for all $X_{u, t}$ and $t \geq 0$.

Proof: The production portfolio and consequently $f_{0}\left(s_{u, v, t}, t\right)$ is specified by $\vec{s}_{u, v, t} \cdot-f_{0}\left(s_{u, v, t}, t\right)$ shows the system cost in a typical peak load pricing framework. These costs and consequently also the set $N_{0}\left(s_{u, v, t}, t\right)=\left\{f_{0}\left(s_{u, v, t}, t\right): s_{u, v, t} \in[0 ; S]\right\}$ are convex (c.f. Steffen and Weber (2012)).

The use of resource $u$ is bound from below by 0 and from above by $h_{u} \cdot \int_{0}^{S} D(s) d s$ or $X_{u, t}$ at each point of time $t \geq 0$. If $f_{u}\left(s_{u, v, t}, t\right)>0$, it fulfills the requirements formulated in Proposition 1 and it is determined by $x_{u, t}=h_{u} \cdot Q_{u, t}\left(s_{w, u, t}^{*}, s_{u, v, t}^{*}\right)$ using Proposition 1. Assume $f_{u}\left(s_{u, v, t}, t\right)$ forms its maximum possible value at time t. If $s_{w, u, t}^{*}$ and $s_{u, v, t}^{*}$ converge, the use of resource $u$ and consequently $f_{u}\left(s_{u, v, t}, t\right)$ continuously approaches 0 . So, $N_{u}\left(s_{u, v, t}, t\right)=\left\{f_{u}\left(s_{u, v, t}, t\right): s_{u, v, t} \in\right.$ $[0 ; S]\}$ with $u=2,3, \cdots, n$ is convex.

Similar conditions apply for $N_{n+1}\left(s_{u, v, t}, t\right)=\left\{f_{n+1}\left(s_{u, v, t}, t\right): s_{u, v, t} \in[0 ; S]\right\}$. Consequently, the Cartesian product $N\left(s_{u, v, t}, t\right)=\left\{\left(f_{0}\left(s_{u, v, t}, t\right)+\gamma, f_{1}\left(s_{u, v, t}, t\right), f_{2}\left(s_{u, v, t}, t\right), \ldots, f_{n+1}\left(s_{u, v, t}\right)\right)\right.$ : $\left.s_{u, v, t} \in[0 ; S], \gamma \leq 0\right\}$ is also convex for all $\left(s_{u, v, t}, t\right)$ and $t \geq 0$.

\section{B.3 Proof of Proposition 3}

The characteristics of an initially below $\bar{Z}$ emission constrained path $s_{u, v, t}^{c}$ are well-defined by (17). The path of the scarcity rents is then specified by (8). Since $Z_{j}<\bar{Z}, \nu_{c, t}=0$ and (9) yields

$$
\lambda_{p, t}=\lambda_{p, 0} \cdot e^{(\rho+\alpha) t},
$$

where $\mathrm{CO}_{2}$-cost rise exponentially.

The final Hotelling path $s_{u, v, t}$, when emissions are no longer constrained, is also defined by 
(17) where $\lambda_{p, t}$ equals zero. If

$$
\begin{gathered}
\left(\zeta_{u}-\zeta_{v}\right)<0 \text { then } s_{u, v, t}^{c}<s_{u, v, t}, \\
\text { and if }\left(\zeta_{u}-\zeta_{v}\right)>0 \text { then } s_{u, v, t}^{c}>s_{u, v, t},
\end{gathered}
$$

for all $t \in[0 ; \infty)$. If the specific emissions are equal, both paths are congruent. Hence, the curves of the control paths $s_{u, v, t}^{c}$ and $s_{u, v, t}$ never cross in the relevant time range $t \in[0, \infty)$. For a continuous Hamiltonian, a in-between time interval of length greater than zero, which cuts both control paths, must connect both of them. Chakravorty et al. (2008) describe multiple potential paths connecting both phases which may result in leaving the ceiling using the cleaner fuel and then filling it up with the dirtier fuel again.

In our approach, during the second phase, $Z_{j}$ must strictly stay at the ceiling: First, consider a single year $t=0$ and neglect any future periods. Since resource or emission constraints cannot materialize in this single period, the optimization problem from (16) reduces to

$$
\max _{s_{u, v}, u, v \in A}-\sum_{A}\left(c_{i n v, u} \cdot K_{u}+c_{o p, u} \cdot Q_{u}\right)
$$

which yields an internal optimality condition for an efficient portfolio that is defined by

$$
s_{u, v}=\frac{c_{i n v, u}-c_{i n v, v}}{c_{o p, v}-c_{o p, u}}
$$

A shift within the portfolio away from this optimality condition is only motivated through an inclusion of future periods. The scarcity of resources is regarded via a rent $\lambda_{u, t}>0$, tight emission targets are considered via $\mathrm{CO}_{2}$-costs $\lambda_{p, t}<0$. This inequality conditions are still applicable after having reached the ceiling and still not on a pure Hotelling path $\left(\theta_{C}<t<\theta_{H}\right)$. But then, $\lambda_{p, t}=0$ would result in a more polluting mix which would break the ceiling. Hence, we pay additional externality or $\mathrm{CO}_{2}$-cost since we have to switch to a less polluting mix, a non-optimal one seen from a pure resource perspective. If we left now the emission ceiling, we would switch to an even less polluting mix which is more expensive as necessary. This would happen in advance of future (less relevant) periods and would waste natural dilution potential. So, it can never be cost-minimal. In a sum, a cost-minimal portfolio will, once reached the ceiling, have to stay there for a non-zero time period until a pure Hotelling path begins.

\section{B.4 Details of selected control paths}

In the following, we describe how the actual control paths can be derived for selected exemplary cases if the technology characteristics, initial endowments and emission targets are known.

\section{B.4.1 Two technologies available and obsolete $\mathrm{CO}_{2}$-restrictions}

If there is a renewable $(u=1)$ and a fossil technology $(u=3)$ with an initial resource stock scarce enough to make a ceiling obsolete, but abundant enough to result in an initial exclusive use of the fossil technology, there are two unknowns $\lambda_{3,0}$ and $\theta_{R}$, with two equations as follows: 
- Cumulative use of resource 3:

$$
h_{3} \cdot\left[\theta_{R} \cdot \int_{0}^{S} D(s) d s+\int_{\theta_{R}}^{\infty} \int_{0}^{s_{1,3, t}} D(s)-D\left(s_{1,3, t}\right) d s d t\right]=X_{3}^{0}
$$

with $s_{1,3, t}=f\left(\lambda_{3,0}, \lambda_{p, 0}=0\right)$

- The renewable technology entering the efficient portfolio:

$$
s_{1,3, \theta_{R}}\left(\lambda_{3,0}, \lambda_{p, 0}=0\right)=S
$$

\section{B.4.2 Two technologies available and relevant $\mathrm{CO}_{2}$-restrictions}

If there is a renewable $(u=1)$ and a fossil technology $(u=3)$ with an initial resource stock abundant enough to make a ceiling relevant, there are five unknowns $\lambda_{3,0}, \lambda_{p, 0}, \theta_{R}, \theta_{C}$ and $\theta_{H}$, with five equations as follows:

- Cumulative use of resource 3 :

$$
\begin{aligned}
& h_{3} \cdot\left[\theta_{R} \cdot \int_{0}^{S} D(s) d s+\int_{\theta_{R}}^{\theta_{C}} \int_{0}^{s_{1,3, t}^{c}} D(s)-D\left(s_{1,3, t}^{c}\right) d s d t\right. \\
+ & \left.\left(\theta_{H}-\theta_{C}\right) \cdot \bar{x}_{3}+\int_{\theta_{H}}^{\infty} \int_{0}^{s_{1,3, t}} D(s)-D\left(s_{1,3, t}\right) d s d t\right]=X_{3}^{0}
\end{aligned}
$$

with $s_{1,3, t}^{c}=f\left(\lambda_{3,0}, \lambda_{p, 0}\right), s_{1,3, t}=f\left(\lambda_{3,0}\right)$ and $\bar{x}_{3}=\alpha / \zeta_{3} \cdot \bar{Z}$

- Continuity of $Z_{j}$

$$
Z_{\theta_{C}}\left(\lambda_{3,0}, \lambda_{p, 0}\right)=\bar{Z} \text { and } Z_{\theta_{H}}\left(\lambda_{3,0}\right)=\bar{Z}
$$

- The renewable technology entering the efficient portfolio:

$$
s_{1,3, \theta_{R}}\left(\lambda_{3,0}, \lambda_{p, 0}\right)=S
$$

- Cumulative emissions

$$
\begin{array}{r}
\zeta_{3} \cdot\left[\theta_{R} \cdot \int_{0}^{S} D(s) d s+\int_{\theta_{R}}^{\theta_{C}} \int_{0}^{s_{1,3, t}^{c}} D(s)-D\left(s_{1,3, t}^{c}\right) d s d t\right] \\
-\int_{0}^{\theta_{C}} \alpha \cdot Z_{t} d t=\bar{Z}-Z_{0}
\end{array}
$$

\section{B.4.3 Three technologies available and relevant $\mathrm{CO}_{2}$-restrictions}

If there is a renewable $(u=1)$, a fossil non-CCS $(u=3)$ and CCS technology $(u=2)$ with a single initial resource stock abundant enough to make a ceiling relevant, and in addition the cost, resource and ceiling characteristics result in CCS entering the portfolio after the renewable, there are seven unknowns $\lambda_{3,0}, \lambda_{p, 0}, \theta_{R}, \theta_{C}, \theta_{H}, \theta_{C C S_{1}}$ and $\theta_{C C S_{2}}$, with seven equations as follows: 
- Cumulative use of resource 3 :

$$
\begin{array}{r}
h_{3} \cdot\left[\theta_{R} \cdot \int_{0}^{S} D(s) d s+\int_{\theta_{R}}^{\theta_{C C S_{1}}} \int_{0}^{s_{1,3, t}^{c}} D(s)-D\left(s_{1,3, t}^{c}\right) d s d t\right. \\
\left.+\left(\theta_{C C S_{2}}-\theta_{C}\right) \cdot \bar{x}_{3}+\int_{\theta_{H}}^{\infty} \int_{0}^{s_{1,3, t}} D(s)-D\left(s_{1,3, t}\right) d s d t\right] \\
+h_{2} \cdot\left[\int_{\theta_{C C S_{1}}}^{\theta_{C C S_{2}}} \int_{0}^{s_{1,2, t}^{c}} D(s)-D\left(s_{1,2, t}^{c}\right) d s d t\right] \\
+\left(h_{3}-h_{2}\right) \cdot\left[\int_{\theta_{C C S_{1}}}^{\theta_{C}} \int_{0}^{s_{2,3, t}^{c}} D(s)-D\left(s_{2,3, t}^{c}\right) d s d t+\left(\theta_{H}-\theta_{C}\right) \cdot \bar{x}_{3}\right. \\
\left.+\int_{\theta_{H}}^{\infty} \int_{0}^{s_{1,3, t}} D(s)-D\left(s_{1,3, t}\right) d s d t\right]=X_{3}^{0}
\end{array}
$$

with $s_{1,3, t}^{c}=f\left(\lambda_{3,0}, \lambda_{p, 0}\right), s_{1,3, t}=f\left(\lambda_{3,0}\right), s_{1,2, t}^{c}=s_{1,2, t}=f\left(\lambda_{3,0}\right)$ and $\bar{x}_{3}=\alpha / \zeta_{3} \cdot \bar{Z}$

- Continuity of $Z_{j}$

$$
Z_{\theta_{C}}\left(\lambda_{3,0}, \lambda_{p, 0}\right)=\bar{Z} \text { and } Z_{\theta_{H}}\left(\lambda_{3,0}\right)=\bar{Z}
$$

- The renewable technology entering the efficient portfolio:

$$
s_{1,3, \theta_{R}}\left(\lambda_{3,0}, \lambda_{p, 0}\right)=S
$$

- The CCS technology entering the efficient portfolio:

$$
s_{1,2, \theta_{C C S_{1}}}^{c}\left(\lambda_{3,0}\right)=s_{1,3, \theta_{C C S_{1}}}^{c}\left(\lambda_{3,0}, \lambda_{p, 0}\right), s_{1,2, \theta_{C C S_{2}}}^{c}\left(\lambda_{3,0}\right)=\bar{s}_{2,3}
$$

with $h_{3} \cdot \int_{0}^{\bar{s}_{2,3}} D(s)-D\left(\bar{s}_{2,3}\right) d s=\alpha \cdot \bar{Z}$

- Cumulative emissions

$$
\begin{array}{r}
\zeta_{3} \cdot\left[\theta_{R} \cdot \int_{0}^{S} D(s) d s+\int_{\theta_{R}}^{\theta_{C C S_{1}}} \int_{0}^{s_{1,3, t}^{c}} D(s)-D\left(s_{1,3, t}^{c}\right) d s d t\right. \\
\left.+\int_{\theta_{C C S_{1}}}^{\theta_{C}} \int_{0}^{s_{2,3, t}^{c}} D(s)-D\left(s_{2,3, t}^{c}\right) d s d t\right]-\int_{0}^{\theta_{C}} \alpha \cdot Z_{t} d t=\bar{Z}-Z_{0}
\end{array}
$$




\section{References}

AG Energiebilanzen e.V., 2012. Bruttostromerzeugung in Deutschland von 1990 bis 2011 nach Energieträgern.

Bohi, D. R., 2010. Analyzing demand behavior: A study of energy elasticities. RFF and Earthscan [distributor], Washington and D.C and London.

Boiteux, M., 1960. Peak-Load Pricing. The Journal of Business 33 (2), 157.

Chakravorty, U., Krulce, D. L., 1994. Heterogeneous Demand and Order of Resource Extraction. Econometrica 62 (6), 1445-1452.

Chakravorty, U., Magné, B., Moreaux, M., 2006. A Hotelling model with a ceiling on the stock of pollution. Journal of Economic Dynamics and Control 30 (12), 2875-2904.

Chakravorty, U., Moreaux, M., Tidball, M., 2008. Ordering the Extraction of Polluting Nonrenewable Resources. American Economic Review 98 (3), 1128-1144.

Chao, H.-p., 1983. Peak Load Pricing and Capacity Planning with Demand and Supply Uncertainty. Bell Journal of Economics 14 (1), 179-190.

Crew, M. A., Kleindorfer, P. R., 1979. Public utility economics. St. Martin's Press, New York.

Eisenack, K., Edenhofer, O., Kalkuhl, M., 2012. Resource rents: The effects of energy taxes and quantity instruments for climate protection: Special Section: Frontiers of Sustainability. Energy Policy 48, 159-166.

Farzin, Y. H., 1992. The Time Path of Scarcity Rent in the Theory of Exhaustible Resources. Economic Journal 102 (413), 813-830.

Herfindahl, O. C., 1967. Depletion and economic theory. Extractive resources and taxation.

Hirshleifer, J., 1958. Peak Loads and Efficient Pricing: Comment. The Quarterly Journal of Economics $72(3), 451-462$.

Hotelling, H., 1931. The economics of exhaustible resources. Journal of Political Economy 39, $137-175$.

İşlegen, Ö., Reichelstein, S., 2011. Carbon Capture by Fossil Fuel Power Plants: An Economic Analysis. Management Science 57 (1), 21-39.

L. S. Pontryagin, V. G. Boltyanskii, R. V. Gamkrelidze, E. F. Mishchenko, 1962. The Mathematical Theory of Optimal Processes. Interscience.

Lafforgue, G., Magné, B., Moreaux, M., 2008. Energy substitutions, climate change and carbon sinks. Ecological Economics 67 (4), 589-597.

Seierstad, A., Sydsæter, K., 1987. Optimal control theory with economic applications. NorthHolland and Sole distributors for the U.S.A. and Canada, Elsevier Science Pub. Co., Amsterdam and and New York and New York and N.Y. and U.S.A. 
Smulders, S., van der Werf, E., 2008. Climate Policy and the Optimal Extraction of High- and Low-Carbon Fossil Fuels. Canadian Journal of Economics 41 (4), 1421-1444.

Steffen, B., Weber, C., 2012. Efficient storage capacity in power systems with thermal and renewable generation. Energy Economics (In Press, Accepted Manuscript).

URL http://dx.doi.org/10.1016/j.bbr.2011.03.031

Steiner, P. O., 1957. Peak Loads and Efficient Pricing. The Quarterly Journal of Economics 71 (4), 585-610.

Stern, N., 2007. The Economics of climate change: Stern review on the economics of climate change. Cambridge University Press, Cambridge.

Stiglitz, J. E., 1976. Monopoly and the Rate of Extraction of Exhaustible Resources. American Economic Review 66 (4), 655.

Sunderkötter, M., Weber, C., 2012. Valuing fuel diversification in power generation capacity planning. Energy Economics 34 (5), 1664-1674.

Tahvonen, O., 1997. Fossil Fuels, Stock Externalities, and Backstop Technology. Canadian Journal of Economics 30 (4), 855-874.

Weber, C., 2005. Uncertainty in the electric power industry: Methods and models for decision support. Springer, New York.

Weinstein, M. C., Zeckhauser, R. J., 1975. THE OPTIMAL CONSUMPTION OF DEPLETABLE NATURAL RESOURCES. Quarterly Journal of Economics 89 (3), 371-392. 